\title{
Research on Command\&Control Simulation System Architecture
}

\author{
Liu Jie ${ }^{\mathrm{a}^{*}}$, Zhang xuemin ${ }^{\mathrm{c}}$, Ge tao ${ }^{\mathrm{d}}$ \\ Department of Equipment Command \&Management \\ Ordnance Engineering College \\ Shijiazhuang, PRC \\ e-mail: too81@sina.com
}

\author{
Sun Haitao \\ Department of Artillery Engineering \\ Ordnance Engineering College \\ Shijiazhuang, PRC \\ e-mail: sunhaitao81@sina.com
}

\begin{abstract}
The Key Problem of Command\&Control Simulation is to solve how to communicate between Command\&Control information and operation models of simulation system. Aiming at such problem, the BML and BOM technical are used together. Meanwhile, both models are presented in XML. Thus the Command\&Control Simulation System Framework is built. At the same time, the basic solution thought for the key problems during the building of the simulation system has been put forward.
\end{abstract}

Keywords- Command\&Control Simulation, Battle Management Language, Base Object Model, Semantic Model

\section{INTRODUCTION}

The key for the success of simulation system in the military field is the interoperation between $\mathrm{C}^{4}$ ISR system and simulation system, including the physical interactions and other data exchanges in any meaningful ways between them. And most of all, the most important interoperation problem is in the Command\&Control field. The most critical C2 information, the commander's intent, his orders and directives, does not currently flow as data. Instead, it is communicated as "free text" elements within messages or as stand-alone files. While suitable for interpersonal communication, it is inadequate for using in simulation system. The free text could not be changed into the useful data for models in the simulation system automatically. So many experts should be used for the conversion from free text to the useful data. Such works are quiet hard and waste time. Meanwhile the data got in that method could only be used in the special simulation system. That means it is quiet hard to use the data in other systems. And it has become the bottle neck for the simulation system development.

To raise the readability of the "free text ", and to realize the interoperation between the command and control system and simulation system, the accurate concept, semantic and rules are needed. To fit the purpose, Battle Management Language (BML) is put forward.

Aiming at raising the interoperation and reusability of the simulation system in the view of model describing language, the command\&control simulation system framework is built in the paper, using BML and Base Object Model (BOM) .

\section{BASIC THOUGHT OF BML AND BOM}

A. $B M L$

BML is the unambiguous language used to command and control forces and equipment conducting military operations and to provide for situational awareness and a shared, common operational picture ${ }^{[1]}$.It originates as the EAGLE Battle management Language in 1990, and the Command and Control Simulation Interface Language (CCSIL $^{[2]}$. BML was taken into research in 1998 by U.S. Army. And the Level 1 Model for Battle Management Language (BML-1) was built in 1999. Till now, there have been many editions. The concept of BML is not only be confined in using of simulated strength or true strength, but also for all the $\mathrm{C} 2$ staff and equipments in the military action.

Aiming at taken $5 \mathrm{Ws}$ (Who, What, When, Where, and Why) as the formal concept, the BML research group was found by SISO in 2004. And BML was pressed as an open standard, called C-BML. In order for BML to provide proper interoperability support, it was determined early on that there should be three different views of the same problem. Conceptually, the three co-supporting views form what has become to be known as the BML triangle. They are BML Doctrine,BML Protocol and BML Representation. Later, the triangle was extended into five views in document ${ }^{[10]}$. They are BML Doctrine,BML Protocol, BML Representation ,BML Ontology and BML Grammar. Standardization efforts in the SISO seek to define this language through three parallel activities: (1) specify a sufficient data model to unambiguously define a set of orders using the Joint Command, Control, and Consultation Information Exchange Data Model (JC3IEDM) as a starting point; (2) develop a formal grammar (lexicon and production rules) to formalize the definition of orders, requests, and reports; (3) develop a formal battle management ontology to enable conceptual interoperability across software systems. This paper focuses on the third activity, development of a formal battle management ontology, by describing an ontology space for potential technical approaches ${ }^{[11]}$.

\section{B. Basic Thought of BOM}

From fall of 1997, the Base Object Model (BOM) was put forward by SISO based on RFOM. The purpose is to 
solve the problem during the development course of FOM, which is the bad reusability and hard repeated work during simulation and modeling. As the basis for the simulation modeling components, $\mathrm{BOM}$, as a group of reusable information productions, is used to express the various patterns in the simulation interactive activities, and to applying in the development of simulation system as the modeling "material". Therefore, the BOM research group was established on 2000 spring SIW meeting by SISO, to study the corresponding method and concepts of BOM in the further. At the same time, the special BOM product development group was built. There has been many documents expressed, such as SISO-STD-003.1-2006 and SISO-STD-003-2006 .Now, BOM has been used as one of the most important technologies for the Extensible Modeling and Simulation Framework, but development build mould and emulation frame ( XMSF ). Most of all, it is also be recognized as one of the key method for the fast development of simulation system.

\section{Main ARChitecture OF SimUlation Training SYSTEM}

\section{A. Relationship of BML Five Views}

The BML five views are BML Doctrine,BML Protocol ,BML Representation ,BML Ontology and BML Grammar. There is not the paratactic relationship of them, but other dependent relationship, as shows in Fig.1.BML Representation is the core for all those views.

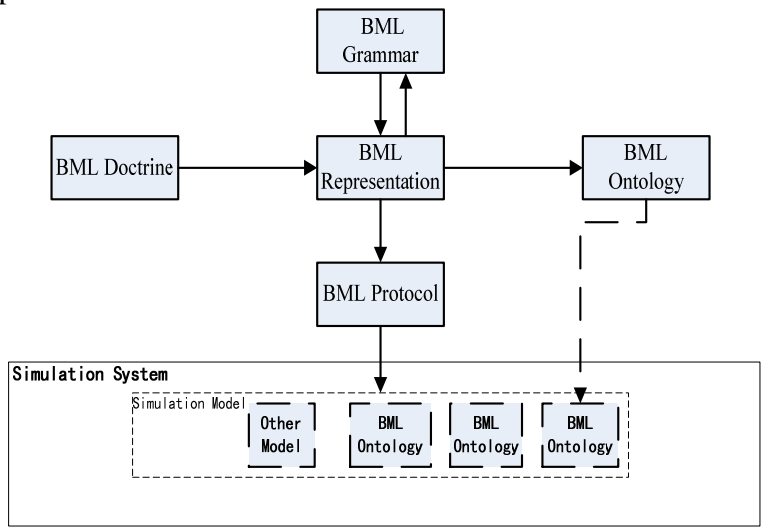

Figure 1 Relationship of BML Five Views

B. Main Architecture for Command\&Control Simulation System

Based on the thought of BML five views, and for the HLA simulation architecture, the BOM model is used to build BML ontology. The thought is shown in Fig.2.

To build a simulation training system, to be honest, the Command\&Control System and Simulation System are built. Meanwhile, the interoperation should be implemented. There are seven parts mainly.

- Human-Computer Interaction Interface

Human-computer interaction interface is one of the most important parts in the human-in-loop simulation system for commander sending command\&control information. Except well human-computer interaction, the form of BML information expression is quite important. As the expression interface will be used for restricting the imports the commander sends to fit BML Grammar. And furthermore, for the computer conversion to the simulation system later.

- Analyse Module for BML Grammar

Analyse Module for BML Grammar is used for the grammar analyse after the $\mathrm{C} 2$ information is imported form Human-Computer Interaction Interface. It is one of the most important mudules for the whole simulation system.

- Building Module for BML Semantic Model

After Grammar analyse, the $\mathrm{C} 2$ information is disassembled into many "meta-phrase". Such meta-phrases could be used as the BML semantic meanings, which are called "BML Semantic Models". The interarrow between Building Module for BML Semantic Model and database of BML Semantic Model, Fig. 2, shows the reusability of the models. That has two meanings: after each grammar analyse, the new meta-phrases will be replenished into the model database. And on the other hand, the semantic models got before can be used in this modeling procedure. To guarantee this reusability, the meta-phrases are described with XML.That database can be recognized as the BML representation.

\section{- $\quad$ Building Module for BOM}

Building Module for BOM is the crucial module for the simulation system. It can be recognized as the BML ontology. Using the database of BML Semantic Model as the foundation, the BOM models can be constructed by the semantic models. For example, some semantic models can be used as the attributes of BOM model.

- Building Module for FOM

Building Module for FOM is used for the assembly of BOM, and forming the FOM for HLA simulation.

- Simulation System

At last, with RTI, and the coded FOM, the simulation system will be built.

\section{Key Problems to Be Solved}

In section3.1, the main architecture of the Command $\&$ Control simulation system is analyzed. And there are three key modules in the architecture to decide the success of the whole system: Analyse Module for BML Grammar, Building Module for BML Semantic Model and Building Module for BOM. They are also the difficult points and core parts for the whole procedure. The realization of them will be analyzed in detail.

\section{A. Analyse for BML Grammar}

Aiming at the actual system development, the C2 information content can be disassembled into $5 \mathrm{Ws}$, which is also the original intention of BML. But such disassembly could not directly be used to distinguish by simulation models and trigger their operations of model. Take order information as example, which includes both TASKER and 
TASKEE. But without other explanation, the computer models could not be able to know which are tasker and also taskee. Therefore if BML is expected to be automatic handled by computer, the formalization grammar is essential.

In his book "Syntactic Structures", published in 1957, Noam Chomsky answered he question "What do we know when we know a language?" by postulating that what we know is a set of words (the lexicon of this language) and a set of rules used to generate sequences of those words (sentences of this language). A sequence of words is defined as grammatical if the sequence can be generated by the rules operating on a lexicon ${ }^{[4]}$.

Chomsky defines four types of grammar. They are ordered within what is designated as a Chomsky hierarchy.

- Grammars of type 0 are unrestricted.

- Grammars of type 1 have rules of the form $\alpha \mathrm{A} \beta \rightarrow$ $\alpha \gamma \beta$, where $\mathrm{A}$ is a non-terminal symbol, $\alpha, \beta$ and $\gamma$ are sequences of terminals and non-terminals, and $\gamma$ consists of at least one symbol. Such a rule can be understood as "A is expanded to $\gamma$ in the context of $\alpha$ and $\beta$ ". Thus, these kinds of grammars are called context sensitive grammars.

- Grammars of type 2 have rules of the form $\mathrm{A} \rightarrow \gamma$, where again $\mathrm{A}$ is a non-terminal symbol and $\gamma$ is a sequence of terminals and non-terminals. Such a rule can be understood as "A is expanded to $\gamma$ ". In contrast to type 1 grammars, no context is to be taken into account. Therefore, these grammars are called context free grammars.

- Grammars of type 3 are even more restricted with respect to their rules. Grammars of type 3 are also called regular grammars.

Grammars of type 0 and type 3 are not used in practical applications and are not considered further in this paper.

Determining a grammar for a language means to find the most restrictive grammar (the higher the type the better) that generates the language. Natural languages are supposed to be context-sensitive as proposed by Chomsky ${ }^{[5]}$. This means that natural languages are supposed to be generated by grammars of type 1. However, BML has to be processed automatically, and the tools (and specific grammars) developed within the field of computational linguistics are restricted to deal with context-free languages, languages generated by grammars of type 2 .

Therefore, the question is, what do we lose if we give BML a type 2 grammar in order to support automatic processing? Here is the answer from a classical workbook on computational linguistics: "The fundamental thing that should be kept in mind is that the overwhelming majority of the structures of any natural language can be elegantly and efficiently parsed using context-free parsing techniques". With this in mind, we choose BML's grammar to be of type 2.

In this subsection, we will evaluate the concept of the $5 \mathrm{Ws}$ and argue for their evolution into the grammar we are defining. If viewed as a formal language, the $5 \mathrm{~W}$ concept could define a grammar in which the Ws (WHO, WHAT, WHERE, WHEN and WHY) make up the set of non- terminal symbols. The production rules of such a grammar would have the form $\mathrm{W} \rightarrow \gamma$ where $\mathrm{W}$ is one of the five $\mathrm{Ws}$ and $\gamma$ is a sequence of terminals based on the BML Semantic models. Thus, a $5 \mathrm{~W}$ grammar would be a type 2 grammar as required, and the Ws would be pre-terminals in the terminology given in BML Grammar.

\section{B. Building for BML Semantic Model Database}

Many BML semantic meta-phrases, or called terminals, form BML semantic model database. Such meta-phrases will be expressed referring to the C2IEDM (Command and Control Information Exchange Data Model ), and detailed in XML.

Take Action for example, Action shows "What" in C2 information. And it has relationship with other metamodels, expressed in XML:

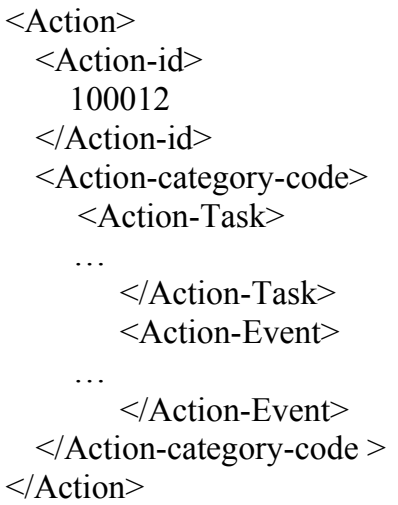

For Action-Task and Action-Event, they can be further expanded according to the basic rules of modeling. Through such procedure for all military C2 information, each type of semantic models will be got.And the database will be perfected continuously.

\section{Mapping between BML Semantic Model and BOM}

To make simulation system operating smoothly, the corresponding models must be built accurately. So the mapping between BML Semantic Model and BOM is the most crucial procedure for the simulation. The BML semantic models are mapped into BOM in two ways. First, BML semantic models is used as the attributes of $\mathrm{BOM}$.That is, the $\mathrm{C} 2$ information is disassembled into semantic models. These models is changed into RTI information, and used by Federate BOM models .Later, the attributes of BOM is changed. Second, BML semantic models are used as the operations of BOM .That is, the $\mathrm{C} 2$ information is disassembled into semantic models. These models are mapped into the operations of BOM in federation.

\section{CONCLUSION}

Aiming at the bottleneck problem of the command \& control simulation system — - the interoperation between command \&control information and simulation models, using BML and BOM, the architecture of the command 
\&control simulation system is designed .Meanwhile the key problems when building the architecture are pointed and the solution thought are put forward. The mapping rules between BML semantic models and BOM is the new research point for the author in the future.

\section{REFERENCES}

[1] Scott A. Carey, Michael R. Hieb.Standardizing Battle Management Language-A Vital Move Towards the Army Transformation[C].01FSIW-067.

[2] Michael Hieb. Extensible Battle Management Language [C], 03FSIW-201.

[3] Huang Zhi. Research on Specified Description and Application of Simulation Scripts [D]. Thesis for the degree of Master, Graduate School of National University of Defense Technology, 2007.

[4] Hopcroft J E, UllmanJD. Itroduction to Automata Theory Languages and Computation[M].Addison-Wesleg, 1979.
[5] Dr. Ulrich Schade, Dr. Michael R. Hieb. Formalizing Battle Management Language A Grammar for Specifying Orders. 06S-SIW068 .

[6] Base Object Model (BOM) Template Specification.[EB/OL].SISOSTD-003.1-2006. [Z].www.siso.org

[7] Guide for Base Object Model (BOM) Use and Implementation. [EB/OL]. SISO-STD-003-2006. [Z].www.siso.org.

[8] B.P.Zeigler. Theory of Modeling and Simulation[M]. John Wiley \& Sons, Inc. 1976.

[9] B.P.Zeigler, Herbert Praehofer, Tag Gon Kim.Theory of Modeling and Simulation : Integrating Discrete Event and Continuous Complex Dynamic Systems. Academic Press. 2000.

[10] Andreas Tolk, Vharles Turnitsa.Battle Management Language: A Triangle with Five Sides[C].06S-SIW-016.

[11] Charles Turnitsa. Filling in the Ontology Space for Coalition Battle Management Language[C].07S-SIW-028.

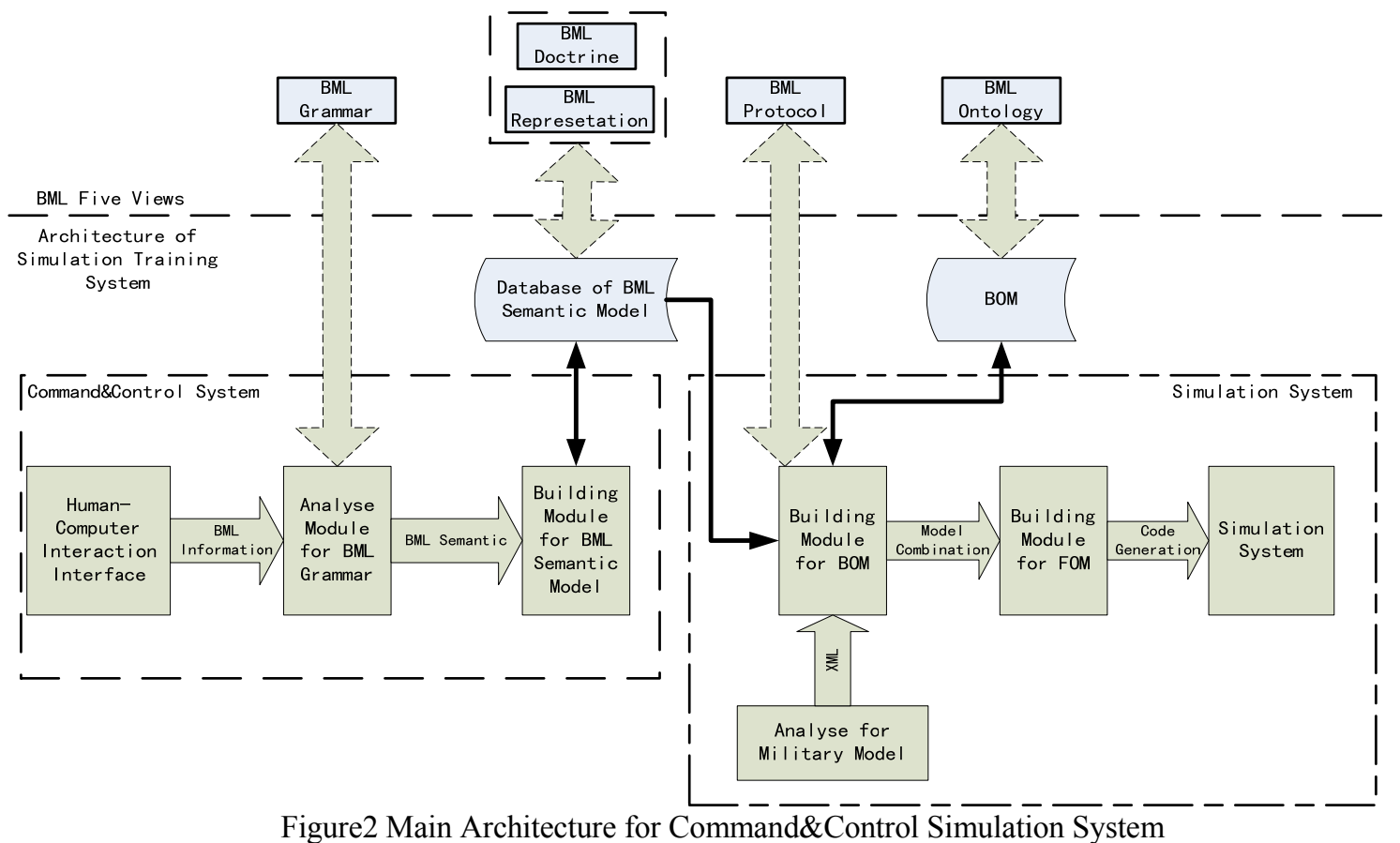

Figure2 Main Architecture for Command\&Control Simulation System 